\title{
A importância da acessibilidade digital na construção de objetos de aprendizagem
}

Prof $^{a}$. Dr ${ }^{a}$. Patricia Alejandra Behar - NUTED/UFRGS - pbehar@terra.com.br

Msc. Eliane Kiss de Souza - PPGIE/UFRGS - elianekiss@ gmail.com

$\mathrm{Msc}^{\text {anda }}$ Camila Guedes Guerra Góes - PPGEDU/UFRGS - milagguerra@ gmail.com

$\mathrm{Msc}^{\text {anda }}$ Edilma Machado de Lima - PPGEDU/UFRGS - edilmalima@gmail.com

Resumo. Este artigo trata sobre a evolução do conceito de acessibilidade digital, abrange reflexões sobre as barreiras de acesso a materiais digitais e ressalta a importância desses conhecimentos com orientações básicas para a construção de objetos de aprendizagem acessível. Apresenta-se, como exemplo, a elaboração de um objeto de aprendizagem para ser ministrado em forma de oficina, mostrando os benefícios que o mesmo pode proporcionar em relação às desigualdades sociais.

Palavras-chave: acessibilidade digital, objeto de aprendizagem acessível, construção de objetos.

\section{The importance of accessibility in the construction of the learning objects}

\begin{abstract}
This article discusses the evolution of the concept of digital accessibility, includes reflections on the barriers for access to digital materials and emphasizes the importance of this knowledge with basic guidelines for the construction of accessible objects of learning. It is presented as example, the preparation of an object of learning to be administered in the form of workshop, showing the benefits that it can provide in relation to social inequalities.
\end{abstract}

Key-words: digital accessibility, object of learning accessible, construction of objects

\section{Introdução}

A acessibilidade é uma das reivindicações dos movimentos realizados por pessoas com deficiências. A partir de um breve histórico desses movimentos compreende-se, na atualidade, a importância da acessibilidade digital à construção de objetos de aprendizagem.

As discussões sobre acessibilidade, no início da década de 80 , giravam em torno da eliminação de barreiras arquitetônicas para pessoas com deficiência, já no final dessa década passou-se a discutir, conjuntamente, a eliminação de barreiras atitudinais (preconceitos). Na década seguinte, incluiu-se na discussão a eliminação de barreiras de comunicação e transporte, contemplando, então, outras deficiências além das limitações motoras. Somente no final dos anos 90 a preocupação passa da eliminação de obstáculos à garantia de acesso, justificando, assim, o uso, junto ao termo acessibilidade, do termo desenho universal ${ }^{1}$.

A partir do novo milênio, surge um novo rumo nas discussões: a garantia de acessibilidade em todas as suas dimensões e para todos os cidadãos. Os movimentos não são mais em torno da eliminação de obstáculos, mas ao direito de ingresso, permanência e usufruto de todos os bens e serviços sociais. Esse novo rumo leva a uma sociedade inclusiva, por isso que, na atualidade, segundo a enciclopédia livre 
Wikipédia, a acessibilidade significa não apenas permitir que pessoas com deficiências participem de atividades que incluem o uso de produtos, serviços e informação, mas a inclusão e extensão do uso destes por todas as parcelas presentes em uma determinada população.

Assim, pensando sobre a escola inclusiva, Werneck (2004) afirma que "A melhor explicação é a da professora Maria Teresa Égler Mantoan, da Unicamp: 'A escola inclusiva nada mais é do que a conseqüência natural de uma escola de qualidade para todos"”.

Levando-se em conta que, na atualidade, a sociedade está a caminho da inclusão e que cada vez mais o acesso de pessoas com necessidades especiais e com mobilidade reduzida a meios e materiais educacionais digitais aumentam, surge o seguinte questionamento: $\mathrm{O}$ que é necessário considerar, em termos de acessibilidade digital, como um conhecimento indispensável à criação de objetos de aprendizagem acessível?

\section{O Conceito de acessibilidade digital}

As discussões, nas décadas de 80 e 90 , em torno da acessibilidade, foram regulamentadas no artigo $8^{\circ}$ do Decreto-lei 5296, de 2 de dezembro de 2004, que regulamenta a Lei de $\mathrm{n}^{\circ} 10.048$, de 8 de novembro de 2000 , que prioriza o atendimento às pessoas com deficiências, e a Lei 10.098, de 19 de dezembro de 2000, que estabelece normas gerais e critérios básicos para a promoção da acessibilidade.

“Artigo $8^{\circ}$ - Para os fins de acessibilidade, considera-se:

I - acessibilidade: condição para utilização, com segurança e autonomia, total ou assistida, dos espaços, mobiliários e equipamentos urbanos, das edificações, dos serviços de transporte e dos dispositivos, sistemas e meios de comunicação e informação, por pessoa portadora de deficiência ou com mobilidade reduzida;

II - barreiras: qualquer entrave ou obstáculo que limite ou impeça o acesso, a liberdade de movimento, a circulação com segurança e a possibilidade de as pessoas se comunicarem ou terem acesso à informação, classificadas em:

a) barreiras urbanísticas: as existentes nas vias públicas e nos espaços de uso público;

b) barreiras nas edificações: as existentes no entorno e interior das edificações de uso público e coletivo e no entorno e nas áreas internas de uso comum nas edificações de uso privado multifamiliar;

c) barreiras nos transportes: as existentes nos serviços de transportes;

$\mathrm{e}$

d) barreiras nas comunicações e informações: qualquer entrave ou obstáculo que dificulte ou impossibilite a expressão ou o recebimento de mensagens por intermédio dos dispositivos, meios ou sistemas de comunicação, sejam ou não de massa, bem como aqueles que dificultem ou impossibilitem o acesso à informação."

No início do milênio o termo acessibilidade se refere ao direito de ingresso, permanência e usufruto de todos os bens e serviços sociais, passando a discussão a girar em torno do conceito de sociedade inclusiva, sendo criado um símbolo internacional para acessibilidade, conforme figura 1.

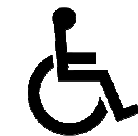

FIGURA 1- Símbolo Internacional para Acessibilidade 
A Resolução 45/91 da ONU, além de estabelecer um conceito de sociedade inclusiva, propôs a construção de uma sociedade para todos até 2010. Para Werneck (2004), "Uma sociedade inclusiva é aquela capaz de contemplar sempre, todas as condições humanas, encontrando meios para que cada cidadão, do mais privilegiado ao mais comprometido, exerça o direito de contribuir com seu melhor talento para o bem comum".

A acessibilidade na web se refere à permissão ao acesso por todos, independente do tipo de usuário, situação ou ferramenta. Portanto, é preciso criar ou tornar as ferramentas e páginas acessíveis para quem as utiliza, sejam pessoas com deficiências ou não, beneficiando, também, idosos, usuários de navegadores alternativos, de tecnologia assistiva ${ }^{2}$ e de acesso móvel. O conteúdo das páginas e as ferramentas devem ser acessíveis porque muitas pessoas podem, total ou parcialmente, não ver, ouvir, mover ou mesmo processar com dificuldade algum tipo de informação.

Que deficiência é necessário se levar em conta quando falamos em acessibilidade digital? De acordo com o Decreto no 3.298, de 20 de dezembro de 1999, é considerada pessoa com deficiência a que se enquadra nas seguintes categorias: deficiência física, deficiência auditiva, deficiência visual e deficiência mental.

A importância de incluir o assunto acessibilidade digital nas discussões da equipe responsável pela criação de objetos de aprendizagem, assim como para Educação a Distância, se deve ao índice de portadores de deficiência que temos no país. De acordo com o censo do IBGE de 2000, no Brasil há cerca de 24,6 milhões de portadores de deficiência, correspondendo a $14,5 \%$ da população brasileira. Aproximadamente $48 \%$ deste total (16,5 milhões) são portadores de algum tipo de deficiência visual. Esses dados justificam as recomendações do W3C (World Wide Web Consortium) que visam permitir que todos possam ter acesso aos websites.

Logo, em informática, programas que provêm acessibilidade são ferramentas ou conjuntos de ferramentas que permitem pessoas com deficiências, as mais variadas, utilizarem os recursos que o computador oferece. Essas ferramentas podem se constituir em leitores de tela para deficientes visuais, teclados virtuais para pessoas com deficiência motora ou com dificuldades de coordenação motora, entre outras.

No que se refere às discussões em torno da acessibilidade digital, para Guilherme de Azambuja Lira (presidente da Acessibilidade Brasil ${ }^{3}$ ), o Brasil está à frente de todos os outros países da América do Sul, mas muito aquém do resto do mundo. Mesmo estando aquém, se desenvolveu o projeto "Da Silva", um site que disponibiliza gratuitamente um avaliador de acessibilidade em português, ideal para desenvolvedores de sites avaliarem seu produto ou que necessitem de informações para torná-lo acessível.

Em relação ao acesso à Informação e Comunicação, se destaca o artigo 47 do Decreto-lei 5296, de 2 de dezembro de 2004, para mostrar a conquista já alcançada em termos de legislação:

“Artigo 47 - No prazo de até doze meses a contar da data de publicação deste Decreto, será obrigatória a acessibilidade nos portais e sítios eletrônicos da administração pública na rede mundial de computadores (Internet), para o uso das pessoas portadoras de deficiência visual, garantindo-lhes o pleno acesso às informações disponíveis.

$\S 2^{\circ}$ Os sítios eletrônicos acessíveis às pessoas portadoras de deficiência conterão símbolo que represente a acessibilidade na rede mundial de computadores, a ser adotado nas respectivas páginas de entrada. 


\section{Barreiras de acesso ao objeto de aprendizagem}

Objeto de aprendizagem (OA), segundo Behar et al (2007, p.2) "são recursos digitais modulares, usados para apoiar a aprendizagem presencial e à distância. Pode ser considerado um OA, qualquer recurso digital que possa ser reutilizado e auxilie na aprendizagem. Logo, pode conter simples elementos como um texto, vídeo, ser um hipertexto, um curso, aplicativo ou até mesmo uma animação com áudio e recursos mais complexos (...) se caracteriza por promover a construção de conhecimento através da interação."

Não há consenso em relação ao conceito de "Objeto de Aprendizagem”, autores o descrevem de diferentes formas. Entre tantas definições, objetos de aprendizagem podem ser definidos como sendo "materiais educacionais com objetivos pedagógicos que servem para apoiar o processo de ensino-aprendizagem". Assim, consideram-se os objetivos que estão propostos para um determinado grupo, para o qual o objeto de aprendizagem foi criado ou re (utilizado), pode-se complementar o conceito com Tarouco et al (2003), que o define como qualquer recurso, suplementar ao processo de aprendizagem, que pode ser reusado para apoiar a aprendizagem. Desta forma, a expressão objeto educacional (learning object) refere-se a materiais educacionais projetados e construídos em pequenos conjuntos com vistas a maximizar as situações de aprendizagem em que o OA pode ser utilizado.

$\mathrm{O}$ que as pessoas com deficiência utilizam para acessar um objeto de aprendizagem? Os que são privados da visão utilizam o leitor de tela ${ }^{4}$, usam navegadores textuais $\mathrm{s}^{5}$ ou navegadores com voz ${ }^{6}$, também fazem uso da tecla "TAB" para navegar somente em links, ao invés de ler todas as palavras que estão na página. Já os que possuem visão subnormal ou baixa visão, usam monitores grandes e aumentam o tamanho das fontes e imagens, os ampliadores de tela ${ }^{7}$. As pessoas com daltonismo apresentam dificuldades na percepção a certas cores, vermelho e verde ou amarelo e azul, por isso, personalizam fontes e cores. As pessoas com deficiência auditiva necessitam de legendas ou imagens suplementares para entender o conteúdo de um objeto de aprendizagem, pois tem como primeira língua, o LIBRAS (Língua Brasileira de Sinais), e nem sempre conseguem fazer uma leitura fluente de outra língua. As pessoas com deficiência física ou motora, em geral usam um mouse especial, um teclado alternativo ${ }^{8}$, um dispositivo tipo ponteira fixado na cabeça ou na boca, software de reconhecimento de voz e outras tecnologias assistivas para acesso e interação. As pessoas com deficiência mental podem necessitar de um leitor de tela com sintetizador de voz e legendas, desativar animações ou sons para focar o conteúdo da página, como também, fixar as imagens por mais tempo para compreender a informação.

Abaixo exemplos de barreiras que as pessoas com deficiência encontram ao acessar um objeto de aprendizagem:

Deficiente visual:

a) vídeos que não possuem descrição textual ou sonora.

b) tabelas que não fazem sentido quando lidas célula por célula ou, em modo linearizado, frames que não possuem a alternativa "noframe", ou que não possuem nomes significativos.

c) formulários que não podem ser navegados em uma sequiência lógica ou que não estão rotulados.

d) navegadores e ferramentas de autoria que não possuem suporte de teclado para todos os comandos. 
e) navegadores e ferramentas de autoria que não utilizam programas de interfaces padronizadas para o sistema operacional em que foram baseados.

f) documentos formatados sem seguir os padrões web que podem dificultar a interpretação por leitores de tela

Baixa visão - páginas:

a) com tamanhos de fontes absoluta, que não podem ser aumentadas ou reduzidas com facilidade.

b) que, devido ao layout inconsistente, são difíceis de navegar quando ampliadas por causa da perda do conteúdo adjacente.

c) que possuem pouco contraste.

d) de textos apresentados como imagens, porque não quebram as linhas quando ampliadas.

Daltonismo:

a) cor é usada como único recurso para enfatizar o texto.

b) contrastes inadequados entre as cores da fonte e fundo.

c) navegadores que não suportam a opção para o usuário utilizar sua própria folha de estilo.

Deficiência auditiva - ausência de:

a) legendas ou transcrições de áudio.

b) imagens suplementares relacionadas, como o conteúdo do texto.

c) de linguagem simples e clara, requisitos para entrada de voz. ou:

d) atividades em que o tempo de utilização é limitado.

e) navegadores e ferramentas que não possuem suporte para teclado alternativo ou botões para todos os comandos efetuados por mouse.

f) formulários que não podem ser navegados com a tecla "TAB" em uma seqüência lógica.

A acessibilidade digital está incluída nas discussões sobre tecnologias assistivas pela importância em relação à educação inclusiva. $\mathrm{Na}$ criação de objetos de aprendizagem todas as ferramentas da plataforma, como correio eletrônico, fóruns de discussão, criação de páginas Web e distribuição de conteúdos, devem levar em conta o acesso igualitário, contemplando, assim, as necessidades específicas das pessoas com deficiências. Para Sá (2004), em especial as universidades públicas deveriam implantar ou desenvolver plataformas que permitam uma educação inclusiva.

Para que a produção desses objetos de aprendizagem não tenha custos elevados, é possível baixar gratuitamente, via Internet, adaptações de hardware ou softwares especiais de acessibilidade com simuladores de teclado e de mouse, varredura e leitores de tela. Outros, segundo Sá (2004), seriam o CD-ROM Kit e os catálogos de ajuda técnica. No Brasil, quanto ao uso de tecnologias assistivas aplicadas à educação de crianças com déficit cognitivo e limitações sensoriais e/ou motoras, destacam-se os programas de "Informática na Educação Especial", como o mantido por uma instituição especializada em Salvador/BA e o NIEE (Núcleo de Informática na Educação Especial) da UFRGS (Universidade Federal do Rio Grande do Sul). Em relação aos softwares simuladores de mouse e teclado, como o "Teclado Amigo" e os softwares para a construção de pranchas de comunicação alternativa "Plaphoons", existe a possibilidade de serem encontrados gratuitamente na Internet. 


\section{A Construção de um objeto de aprendizagem acessível}

A construção de um objeto requer alguns passos iniciais importantes, como qualquer projeto. Deve-se ter em mente que o planejamento é o primeiro passo para a construção, bem como, claros os objetivos a serem alcançados e para qual público alvo está sendo dirigido o trabalho. A composição de uma equipe de profissionais com conhecimento técnico e pedagógico, em um trabalho interdisciplinar, é essencial para alcançar resultados positivos, tanto em relação à construção como a utilização posterior do objeto de aprendizagem.

No que se refere à construção do objeto, é requerido também dos desenvolvedores um conhecimento sobre as barreiras que impossibilitam aos usuários, com alguma limitação, o acesso ao mesmo. É necessário ter presente que o uso de alguns recursos visuais, como tabelas ou programas como o flash, limitará o leitor de tela a fornecer todas as informações contidas no objeto para o usuário. Da mesma forma que o uso de uma linguagem de estilo, no desenvolvimento deste objeto, irá proporcionar tanto condições de leitura, pelos programas, como um layout perfeito.

Segundo Amaral (2006), o CSS (Cascading Style Sheets) é uma linguagem de estilo utilizada para descrever a apresentação de um documento escrito em uma linguagem de marcação, tais como HTML, XHTML ou XML. Essas definições são aplicadas a documentos de diversas maneiras, alterando a forma como as informações são apresentadas. As formatações do CSS são normalmente aplicadas aos elementos (tags) do HTML.

O responsável pela definição das especificações do CSS é o W3C, uma organização não-governamental responsável pela definição de padrões de diversas tecnologias relativas à internet, como o HTML, o protocolo HTTP e o XML. É formada por membros de diversas empresas, entre elas a Apple, Microsoft e Netscape.

Com essa postura diante da construção de objetos de aprendizagem, ou qualquer outro material para meios eletrônicos, é possível proporcionar a democratização da informação e a garantia do pleno exercício da cidadania e a integração social dos cidadãos com alguma limitação ou com necessidades especiais.

\section{O objeto de aprendizagem acessibilidade digital}

A construção do objeto, conforme figura 2, nasceu da proposta de uma disciplina do PPGIE da UFRGS, no primeiro semestre de 2008 e tinha como foco o desenvolvimento de objetos de aprendizagem com a finalidade de serem ministrados em forma de oficinas, como mostra a figura 3. De acordo com Behar (2008a) "A disciplina Oficinas Virtuais de Aprendizagem é composta por um conjunto de Objetos de Aprendizagem desenvolvidos pela equipe do NUTED com a finalidade de serem utilizados em Instituições de Ensino".

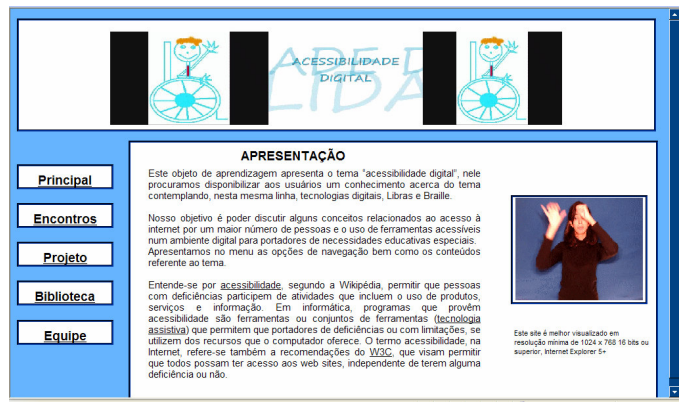

FIGURA 2 - OA acessibilidade digital

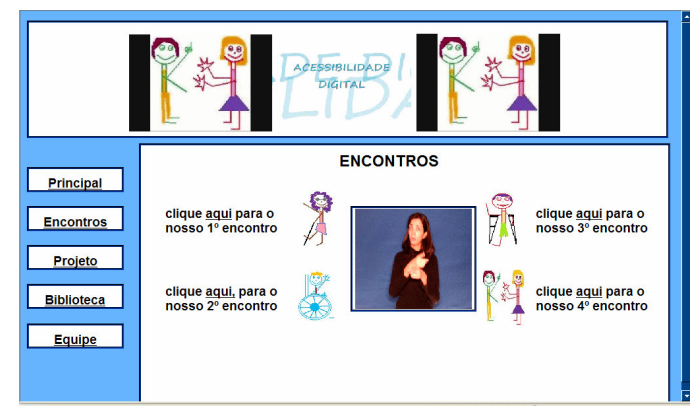

FIGURA 3 - Apresentação das oficinas 
Ainda segundo Behar (2008b), "As oficinas podem ser consideradas, dentro de uma dimensão simbólica, como um 'espaço', um 'lugar' ou um 'ambiente', que objetiva aproximar da realidade dos alunos e favorecer a construção da subjetividade, afastada de uma concepção paternalista e gerencial. Essa perspectiva implica que o papel da escola não é somente ensinar conteúdos, nem vincular a instrução com aprendizagem. Logo, este espaço/ambiente poderia ser pensado como um objeto de aprendizagem que pode ser considerado desde um simples hipertexto, aplicativo ou até em forma de animação. Ou seja, esta estará totalmente integrada com a de Construção de Objetos de Aprendizagem, onde será iniciada a elaboração da Oficina".

O objeto de aprendizagem foi construído durante a disciplina, a partir de três etapas: concepção, planificação e implementação. Na primeira foram definidas as linhas mestras do objeto, público-alvo, as concepções teórico/práticas, entre outros aspectos. $\mathrm{Na}$ segunda etapa, a planificação foi desenvolvido no storyboard ${ }^{9}$ (designer do objeto), mais conhecido como mapa conceitual, conforme figura 4. Neste roteiro foram definidos os objetivos, estratégias metodológicas, avaliação, público alvo, a forma como se desenvolveriam os encontros, recursos a serem utilizados, entre outros componentes.

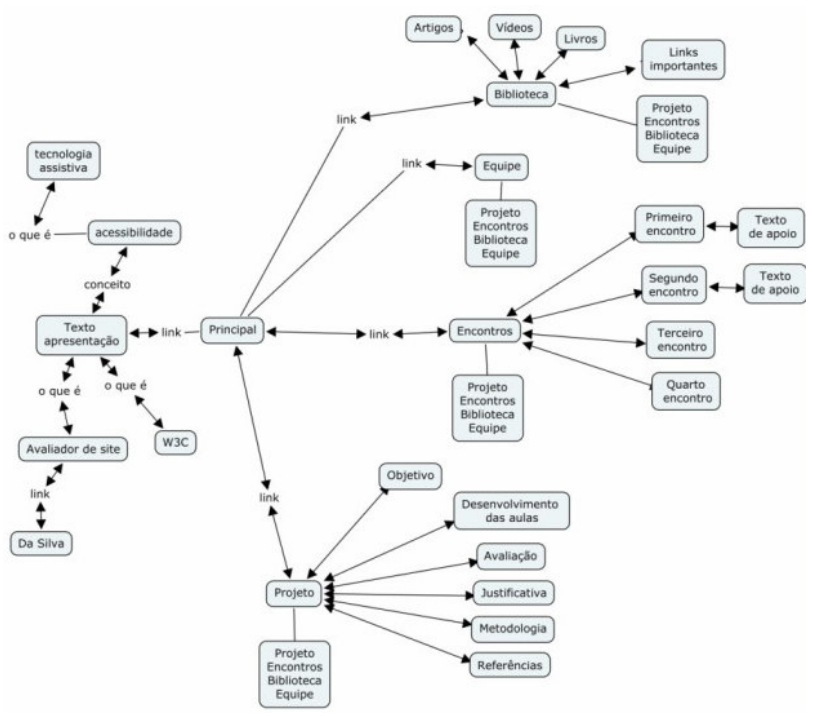

FIGURA 4 - Storyboard do Objeto de Aprendizagem Acessibilidade Digital

O objeto foi idealizado dentro do tema acessibilidade digital, contemplando também as tecnologias assistivas, o LIBRAS e o Braille, duas formas de comunicação usadas por pessoas com necessidades especiais. Todas as páginas principais do objeto dispõem de vídeo com a tradução em Libras conforme a figura 5. Tomou-se como hipótese que quanto mais conhecimentos os professores e educadores tenham sobre estes assuntos, mais condições terão de buscar ajuda e formação para minimizar as desigualdades sociais e chegar-se a uma escola inclusiva ideal. Através de pesquisas na internet e no próprio histórico da disciplina, se constatou que não haviam materiais elaborados dentro deste assunto. 


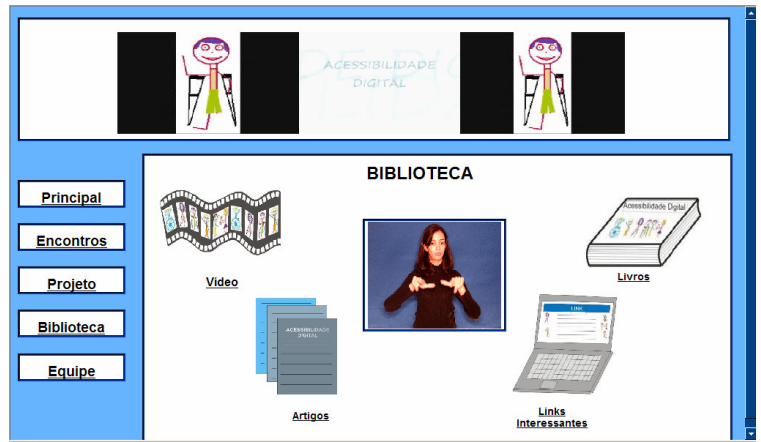

FIGURA 5 - Vídeo de tradução em Libras

Para a terceira etapa, a implementação, a opção foi pelo programa Front Page e o estilo CSS, por se entender que, ao propor o tema acessibilidade, o objeto deveria que estar condizente com essa filosofia, ou seja, ser acessível a todos. Optou-se por imagens animadas e não animadas em formato GIF (Graphics Interchange Format), criadas por uma das pesquisadoras, como mostra a figura 6.

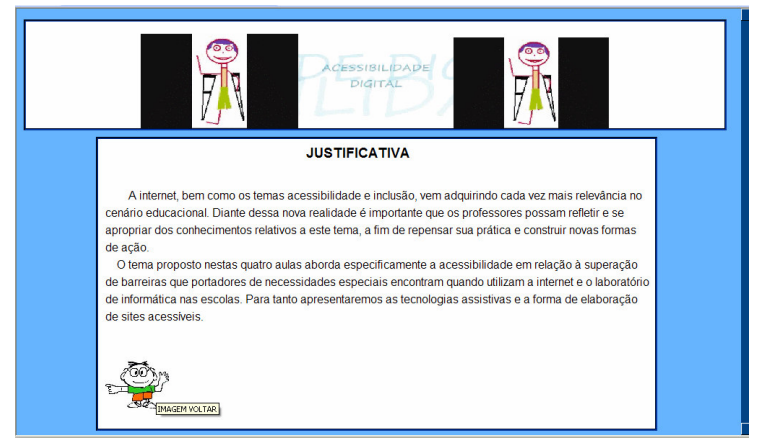

FIGURA 6 - GIFs animados e não animados do OA

O objeto encontra-se disponível em https://www.ead.ufrgs.br/rooda/webfolio/ abrirArquivo.php//Producoes/493/Objeto\%20Trabalho\%20Final/index.htm.

A seguir, são citadas algumas dicas, resultados da experiência e pesquisas da equipe (que tem como membro um usuário com necessidades especiais), para designers e/ou professores em geral que se disponham a construir objetos acessíveis:

- usar o atributo ALT para descrever a função de cada elemento visual ( em imagens e animações);

- incluir legendas e transcrições para o áudio e descrições para o vídeo (multimídia);

- utilizar textos que façam sentido fora do contexto (hiperlinks);

- na organização da página, usar cabeçalhos, listas e uma estrutura consistente;

- é recomendável o uso CSS para layout e estilo, sempre que possível;

- em relação a gráficos e diagramas, sumarizar o conteúdo ou usar o atributo longdesc;

- fornecer conteúdo alternativo aos scripts, applets e plug-ins, para o caso de estarem desabilitados ou de não serem suportados pelo browser;

- em frames, usar o tag e empregar títulos significativos;

- tornar as tabelas compreensíveis à leitura linha a linha, com um resumo ou optar por caixas;

- submeter o material ao avaliador de acessibilidade; e

- validar o trabalho usando ferramentas, checklist e os guias disponíveis em http://www.w3.org/TR/WCAG 


\section{Considerações finais}

Ao encaminhar esta pesquisa para suas considerações finais e tecer alguns comentários, pode-se afirmar que esta é apenas uma etapa, o início de uma trajetória onde muitas descobertas ainda estão por vir, pois, hoje, caminha-se rumo a uma sociedade inclusiva, onde todos devem ter os mesmos direitos de acesso à informação e a uma educação de qualidade.

Neste sentido se acredita que, ao disponibilizar informações sobre a importância da acessibilidade em meio digital e sobre as Tecnologias adaptativa/assistivas, temas já consolidados na legislação, no que se refere à construção de objetos de aprendizagem, se está colaborando para uma sociedade mais justa e igualitária para todos.

Foi aproveitada a oportunidade, na elaboração do objeto de aprendizagem, para colocar em discussão a acessibilidade digital. Porém, ainda não se implementou uma oficina prática, onde seria possível coletar dados significativos para apontar soluções que poderiam auxiliar na construção de objetos.

Considera-se que tanto os conhecimentos sobre acessibilidade como as tecnologias assistivas são fundamentais aos desenvolvedores desses materiais, como, também, para os organizadores de cursos na modalidade de Educação a Distância e educadores do ensino presencial. Assim, foram destacados os conceitos, as conquistas já alcançadas na forma de legislação, as barreiras encontradas pelas pessoas com necessidades especiais, os padrões estabelecidos pelo W3C e as especificações do CSS.

Concluindo, na construção de objetos de aprendizagem é preciso considerar, fundamentalmente, que o acesso a meios e materiais digitais é um direito de todos. Partindo deste princípio, os conhecimentos sobre este tema se tornam indispensáveis.

\section{Notas de Texto}

${ }^{1}$ Conceito de desenho universal, um planejamento arquitetônico ambiental, de comunicação e de transporte onde todas as características das pessoas são atendidas, independentemente de possuírem ou não uma deficiência.

${ }^{2}$ A tecnologia assistiva pode ser compreendida como resolução de problemas funcionais, tendo como perspectiva o desenvolvimento das potencialidades humanas, valorização de desejos, habilidades, expectativas positivas e da qualidade de vida. Sá (2000)

3 Suas principais ações são voltadas para a disseminação das normas nacionais e internacionais de acessibilidade e o desenvolvimento de conteúdos de interesse específico para a área de deficiência.

${ }^{4}$ Software que lê o texto que está na tela do microcomputador e a saída desta informação é através de um sintetizador de voz ou um display Braille.

${ }^{5}$ Navegador baseado em texto, diferente dos navegadores com interface gráfica onde as imagens são carregadas.

${ }^{6}$ Sistema que permite a navegação orientada pela voz.

${ }^{7}$ Software que amplia o conteúdo da página para facilitar a leitura.

8 Dispositivo de hardware ou software usado por pessoas com deficiência física, fornece um modo alternativo de dispor as tecla.

${ }^{9}$ Storyboard é o desenvolvimento de um roteiro, para visualização de algo que será realizado em outro meio.

\section{Referências Bibliográficas}

ACESSIBILIDADE. Disponível em: http://pt.wikipedia.org/wiki/Acessibilidade. Acesso em: 9/06/2008

ACESSIBILIDADE BRASIL. Disponível em: http://www.acessobrasil.org.br/. Acesso em: 9/06/2008 
ACESSIBILIDADE NA WEB. Disponível em: http://www.serpro.gov.br/acessibilidade/g_recom.php. Acesso em: 9/06/2008

ACESSIBILIDADE NO MUNDO DO TRABALHO. Disponível em: http://www.sorri.com.br/ artigos/AcessibilidadeNoMundoDoTrabalho.pdf. Acesso em: 10/06/2008

AMARAL, L. G. Css - Cascading Style Sheets - Guia de Consulta Rápida - 2a Edição 2006

BEHAR,P. A. (2008a) OFICINAS. Disponível em: http://homer.nuted.edu.ufrgs.br/oficinas_2008/. Acesso em: 04/04/2008

BEHAR, P. A. (2008b) CONSTRUÇÃO DE OFICINAS VIRTUAIS. Disponível em: http://homer. nuted.edu.ufrgs.br/oficinas_2008/oficinas/index.htm. Acesso em: 10/06/2008

BEHAR, P. A. ; GASPAR M. I. Uma perspectiva curricular com base em objetos de aprendizagem. In: Virtual Educa 2007, 2007, São José dos Campos. Disponível em: http://ihm.ccadet.unam.mx/ virtualeduca2007/pdf/37-PB.pdf, acesso em: 17/10/2008.

BRASIL. DECRETO No 3.298, de 20 de dezembro de 1999. Disponível em: http://br.vlex.com/ $\mathrm{vid} / 34331882$. Acesso em: 10/06/2008

BRASIL.DECRETO-LEI 5296, de 2 de dezembro de 2004. Disponível em: http://www.planalto.gov. br/ccivil/_ato2004-2006/2004/decreto/d5296.htm. Acesso em: 06/06/2008

CENSO 2002. Ministério do planejamento, orçamento e gestão. IBGE. Disponível em http://www.ibge.gov.br/home/presidencia/noticias/noticia_visualiza.php?id_noticia=438\&id_pagina=1. Acesso em: 06//06/2008

DESENHOS ANIMADOS - ROTEIRO, STORYBOARD E PERSONAGENS - Erick Souza. Disponível em: http://blog.ericksouza.com/roteiro-storyboard-e-personagens/ Acesso em: 17/10/2008

DEFICIÊNCIA AUDITIVA. Disponível em: http://www010.dataprev.gov.br/sislex/paginas/23/1999/ 3298.htm. Acesso em: 9/10/2008

DICAS RÁPIDAS PARA CONSTRUIR WEB SITES ACESSÍVEIS, Brazilian Portugese translation of the WAI QuickTips. Disponível em: http://www.w3.org/WAI/quicktips/qt.br.htm. Acesso em: $08 / 06 / 2008$

EXEMPLO DE BARREIRAS AO ACESSAR PÁGINAS E USO DE TECNOLOGIA ASSISTIVA. Disponível em: http://www.serpro.gov.br/acessibilidade/acesso.php. Acesso em: 10/06/2008

WERNECK. C (2004) INCLUSÃO SOCIAL - TODOS SOMOS RESPONSÁ VEIS. Disponível em: http://www.cidadaniaembraer.org.br/site/pagina.php?idconteudo=209\&entrevistas AnterioresPage $=4$. Acesso em: 20/05/2008.

MATERIAL PEDAGÓgICO E TECNOLOGIAS ASSISTIVAS. SÁ. E. D. (2004). Disponível em: http://www.acessibilidade.net/at/kit2004/Programas\%20CD/ATs/cnotinfor/Relatorio_Inclusiva/pdf/Tecno logias_assistivas_pt.pdf. Acesso em: 10/05/2008

OBJETO DE APRENDIZAGEM. Disponível em: http://www.rived.mec.gov.br/site_objeto_ lis.php. Acesso em: 9/10/2008

RECOMENDAÇÕES W3C. Disponível em: http://www.w3c.br/. Acesso em: 05/05/2008

RESOLUÇÃO ONU N.o 45/91.14, de Dezembro de 1990. Disponível em: http://app.crea-rj.org.br/ portalcreav2midia/documentos/resolucaoonu45.pdf. Acesso em: 10/06/2008

TAROUCO, L.M.R.; FABRE, M.-C. J. M.; et al. Reusabilidade de objetos educacionais. Novas Tecnologias na Educação, Porto Alegre, v.1, n.1, p.1-11. 2003. Disponível em: http://www.cinted. ufrgs.br/renote/fev2003/artigos/marie_reusabilidade.pdf. Acesso em: 10/10/2008.

TAROUCO, L. M. R.; FABRE, M. C. J. M.; GRANDO A. R. S.; KONRATH, M. L. P. Objetos de Aprendizagem para M-Learning. In: Congresso Nacional de Tecnologia da Informação e Comunicação, 2004. Florianópolis: SUCESU 